\title{
Influence of bovine serum albumin and fetal bovine serum supplementation during in vitro maturation on lipid and mitochondrial behaviour in oocytes and lipid accumulation in bovine embryos
}

\author{
Maite del Collado A,B,F, Naiara Z. Saraiva C, Flavia L. Lopes ${ }^{\mathrm{D}}$, \\ Roberta C. Gaspar ${ }^{\mathrm{B}}$, Luciana C. Padilha ${ }^{\mathrm{B}}$, Roberta R. Costa', \\ Guilherme F. Rossi ${ }^{\mathrm{B}}$, Roberta Vantini ${ }^{\mathrm{B}}$ and Joaquim M. Garcia ${ }^{\mathrm{B}}$ \\ A Departamento de Medicina Veterinária, Faculdade de Zootecnia e Engenharia de Alimentos, \\ USP, Avenida Duque de Caxias Norte, 225, 13635-900, Pirassununga, SP, Brazil. \\ ${ }^{B}$ Departamento de Medicina Veterinária Preventiva e Reprodução Animal, Faculdade de Ciências \\ Agrárias e Veterinárias, UNESP, Via de Acesso Prof. Paulo D. Castellane SN, 14884-900, \\ Jaboticabal, SP, Brazil.

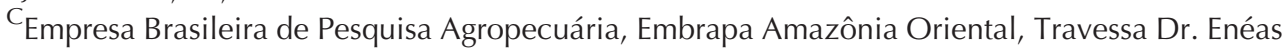 \\ Pinheiro s/n, 66095-100, Belém, PA, Brazil. \\ Departamento de Apoio, Produção e Saúde Animal, Faculdade de Medicina Veterinária de \\ Araçatuba, UNESP, Rua Clóvis Pestana, 793, 16050-680, Araçatuba, SP, Brazil. \\ ${ }^{E}$ Departamento de Biologia Celular e Molecular e de Bioagentes Patogênicos, \\ Faculdade de Medicina de Ribeirão Preto, USP, Avenida Bandeirantes, 3900, \\ 14049-900, Ribeirão Preto, SP, Brazil. \\ ${ }^{\mathrm{F}}$ Corresponding author. Email: delcollado@hotmail.com
}

\begin{abstract}
Proper oocyte maturation is crucial for subsequent embryo development; however, oocyte mitochondrial and lipid-droplet behaviour are still poorly understood. Although excessive lipid accumulation during in vitro production (IVP) of bovine embryos has been linked with impaired cryotolerance, lipid oxidation is essential for adequate energy supply. Fetal bovine serum (FBS) and bovine serum albumin (BSA) are supplements used during IVP, containing high and low lipid content, respectively. This study aimed to understand how these supplements influence oocyte mitochondrial and lipid behaviour during in vitro maturation (IVM) in comparison to in vivo maturation, as well as their influence on development rates and embryo lipid accumulation during IVP. We demonstrate that only in vivo-matured oocytes maintained correlation between lipid content and active mitochondria. IVM media containing FBS increased total lipid content 18 -fold and resulted in higher lipid accumulation in oocytes when compared with media with BSA. IVM using a lower FBS concentration combined with BSA resulted in satisfactory maturation and embryo development and also reduced lipid accumulation in blastocysts. In conclusion, IVM causes changes in mitochondrial and lipid dynamics, which may have negative effects on oocyte development rates and embryo lipid accumulation. Moreover, decreasing FBS concentrations during IVM may reduce embryo lipid accumulation without affecting production rates.
\end{abstract}

Additional keywords: in vitro production (IVP), lipid droplet, mitochondria, reproduction.

Received 19 February 2015, accepted 2 April 2015, published online 19 May 2015

\section{Introduction}

Oocyte maturation is one of the most precise determinants of subsequent embryo quality. During this phase, the oocyte undergoes processes that are crucial not only to activation of the embryonic genome, but also throughout development to the blastocyst stage, given that the female gamete is the sole cytoplasmic donor during the formation of the zygote (Gilbert 2003).

In vitro production (IVP) of cattle embryos has increased considerably in recent years. According to the International Embryo Transfer Society (IETS 2012), in 2011 global rates of in vivo embryo transfers remained stable, while transfers of 
in vitro-produced bovine embryos increased for the sixth consecutive year, placing this reproductive biotechnology as an important player in the cattle industry. However, there are still many limitations to the technology, where only $40-50 \%$ of oocytes, matured and fertilised in vitro, reach the blastocyst stage (Rizos et al. 2002). Furthermore, excessive lipid accumulation occurs following IVP in bovine embryos when compared with in vivo production (Abd El Razek et al. 2000; Rizos et al. 2002), mainly following the use of fetal bovine serum (FBS) in the culture media, which has been associated with low embryo cryotolerance (Rizos et al. 2002, 2003).

The most abundant lipid in oocytes and embryos is triacylglyceride (TG; Ferguson and Leese 1999), which is stored in cytoplasmic lipid droplets. TG is synthesised in endoplasmic reticulum (ER) where the enzyme diacylglyceride acyltransferase 2 (DGAT2) is responsible for the formation of TG from diacylglycerol and acyl-CoA, during the final step of synthesis (Stone et al. 2009). Once synthesised, TGs can be metabolised or accumulated in lipid droplets, and DGAT2 is associated with entry of TG into the formed droplets (Kuerschner et al. 2008). Pirilipin 2 (PLIN2) is a structural protein found on the surface of lipid droplets that is involved in limiting the interaction between lipase and the droplets and, therefore, is positively correlated with their number and size (Bickel et al. 2009). Despite the known deleterious effects on cryopreservation (Rizos et al. 2002), lipids are required by all cells, given that the metabolism of fatty acids in mitochondrial $\beta$-oxidation is one of the most important pathways supplying energy to the cell, in the form of ATP (Cummin 2004).

The importance of mitochondrial oxidation in embryo development is well established and is associated with meiosis reactivation in oocytes, as well as embryo development to the blastocyst stage (Dunning et al. 2010). Although the increase of mitochondrial activity during bovine embryo development was previously described (Tarazona et al. 2006), it is believed that mitochondria of oocyte origin are responsible for energy supply during development, since there is no synthesis of new organelles until the blastocyst stage (Van Blerkom 2011). Although there is a consensus regarding the increase in mitochondrial activity during in vitro maturation (IVM) in different species (Tarazona et al. 2006; Romek et al. 2011), lipid behaviour and the interaction with mitochondria, at this stage, remain poorly understood. Recently, studies have shown an increase of lipid, particularly TG, in the oocyte during bovine IVM, with or without FBS in the maturation media (Ferreira et al. 2008; Aardema et al. 2011; González-Serrano et al. 2013). Migration patterns for lipid and mitochondria have not been intensively investigated, and current knowledge, based on earlier studies with electron microscopy data, shows that during in vivo maturation of bovine oocytes, mitochondrial and lipid migration occur concurrently from the periphery of the cytoplasm to a more dispersed distribution (Kruip et al. 1983; Hyttel et al. 1986).

Considering the variations observed between in vivo- and in vitro-matured bovine oocytes as well as differences in development rates between in vivo- and in vitro-produced blastocysts, a thorough characterisation of the maturation process, both in vivo and in vitro, is warranted. We hypothesised that IVM leads to different lipid and mitochondrial behaviour patterns in matured oocytes compared with the in vivo maturation process, which can, in turn, alter lipid metabolism in in vitro-produced bovine embryos. Based on this hypothesis, the present study aimed to investigate how supplements, with either a high or low lipids content, affect lipid and mitochondrial behaviour in in vitro-matured oocytes, while also making comparisons with the patterns observed during in vivo maturation, and the consequences of this on in vitro embryo development and lipid quantity. To our knowledge, this study is the first to describe the differences between bovine in vitro and in vivo maturation in relation to lipid and mitochondrial migration and quantity. We also demonstrate that it is possible to decrease embryo lipid accumulation by reducing the FBS concentration currently used during IVM in cattle.

\section{Materials and methods}

Reagents were purchased from Sigma Chemical Co. (St. Louis, MO, USA) unless otherwise indicated.

\section{Ethics}

All animal handling was approved by the ethics committee for animal welfare of the Faculdade de Ciências Agrárias e Veterinárias of the Universidade Estadual Paulista 'Júlio de Mesquita Filho' (FCAV/UNESP).

\section{Experimental design}

Three experimental groups with different protein supplements in IVM medium were utilised. The first group was supplemented with $8 \mathrm{mg} \mathrm{mL}^{-1}$ bovine serum albumin (BSA)-fatty acid-free (BSA-FAF; BSA group), the second with 10\% FBS (Cripion; Industria Brasileira, Andradina, Brazil; FBS group) and a third group, called $\mathrm{B}+\mathrm{F}$, contained lower concentrations of each supplement: $6 \mathrm{mg} \mathrm{mL}^{-1} \mathrm{BSA}-\mathrm{FAF}+5 \% \mathrm{FBS}$.

In order to characterise the influence of each maturation medium on oocyte and embryo lipid accumulation during the in vitro process, we calculated the total lipid content of the media used, based on the lipid content of BSA-FAF provided by the manufacturer and by analysing that of FBS using gas chromatography.

Next, we assessed the effects of the different supplements used during IVM on nuclear maturation and migration of cortical granules. Subsequently, we performed a comparative study of the lipid-mitochondrial behaviour between IVM groups and an in vivo-matured group. We also evaluated the effects of IVM treatments on cleavage and blastocyst rates and embryo lipid accumulation. Lastly, to better understand the effects of protein supplementation on lipid metabolism, we investigated the expression of two genes involved in lipid synthesis, PLIN2 and DGAT2, in cumulus cells, in immature and mature denuded oocytes, as well as in embryos.

\section{Quantification and analysis of lipid profile in FBS and calculation of lipid content in IVM media}

The lipid quantification and fatty-acid profile of FBS were performed by gas chromatography. The results of the lipid 
content and fatty-acid percentages were determined by averaging five replicates from samples of $5 \mathrm{~mL}$ of FBS. Following lipid extraction with acid hydrolysis and transformation of acids into fatty-acid methyl esters, the samples were injected into a gas chromatograph (GC Schimadzu 17 A/Class CG 10; GC Shimadzu 17A/Class CG 10, Kyoto, Japan) in a chromatographic column of SP-2560 fused silica $100 \mathrm{~m}$ and $0.25 \mathrm{~mm}$ internal diameter (i.d.). The results of fatty-acid profiles for the samples are expressed in $\mathrm{mg}$ per $100 \mathrm{~mL}$ and $\%$ of area, and the determination of total lipids, calculated from the triglyceride tridecanoic acid (internal standard), is shown in $g$ per $100 \mathrm{~mL}$.

After determination of lipid quantity for FBS (g per $100 \mathrm{~mL}$ ), and taking into account the manufacturer's indication of the maximum lipid content of BSA-FAF ( $0.02 \%$ fatty acids), we calculated the amount of lipid present in each IVM medium, according to the concentration of each supplement used.

\section{Obtaining immature and in vitro- and in vivo-matured oocytes}

We collected cumulus-oocyte complexes (COCs) by follicular aspiration from slaughterhouse ovaries to obtain immature and also to produce in vitro matured oocytes, following IVM. Grade I and II COCs, following the classification described by Leibfried-Rutledge et al. (1987), were selected. A portion of the COCs was randomly allocated to evaluations involving immature oocytes and the remainder were used for IVM in the different maturation media (treatment groups: BSA, FBS and $\mathrm{B}+\mathrm{F})$. The stock for IVM media comprised TCM199 medium (GIBCO BRL, Grand Island, NY, USA) supplemented with $25 \mathrm{mM}$ sodium bicarbonate, $1 \mu \mathrm{g} \mathrm{mL}^{-1} \mathrm{FSH}$ (Folltropin; Bioniche Animal Health, Belleville, ON, Canada), $50 \mu \mathrm{g} \mathrm{mL}^{-1}$ human chorionic gonadotrophin (hCG, Vetecorn; Intervet, Itagaçaba Cruzeiro, Brazil), $1 \mu \mathrm{g} \mathrm{mL}^{-1}$ oestradiol, $83.4 \mathrm{mg} \mathrm{mL}^{-1}$ amikacin (Biochimico Institute, Rio de Janeiro, Brazil) and $0.2 \mathrm{mM}$ sodium pyruvate. For IVM, we maintained COCs at $38.5^{\circ} \mathrm{C}$ and $5 \% \mathrm{CO}_{2}$ in air and high humidity for $24 \mathrm{~h}$.

To collect in vivo-matured oocytes, six crossbred cows were synchronised and overstimulated according to the protocol described in Fig. 1. On the Day 1 of the protocol, we performed transvaginal ultrasound-guided ovum pick-up (OPU) for synchronisation of follicular waves and inserted a progesterone implant (Sincrogest; Ourofino Agribusiness, Cravinhos, Brazil) that remained in place for 7 days. On the Day 4 we began follicular overstimulation by injecting decreasing doses of FSH
(Pluset; Laboratorio Calier SA, Barcelona, Spain) for a total of 250 IU FSH per animal by the Day 7. We applied a single dose of $2 \mathrm{~mL}$ prostaglandin F2alpha (PGF2a) (Sincrocio; Ourofino) on the afternoon of Day 6 and $2 \mathrm{~mL}$ of gonadotrophin-releasing hormone 1 (GnRHl, Sincroforte; Ourofino) to each animal $24 \mathrm{~h}$ before oocyte collection on Day 9.

\section{In vitro production of blastocysts from oocytes undergoing IVM}

Matured oocytes from all three experimental groups (total of 1232, 844 and 959 oocytes for the groups BSA, FBS and B+F, respectively) were used for in vitro fertilisation (IVF) in order to produce blastocysts from the different maturation media. After IVF on Tyrode/albumin/sodium lactate/sodium pyruvate (IVF-TALP) medium supplemented with $0.6 \%$ BSA, zygotes from all experimental groups were cultured in vitro (IVC) for 8 days in synthetic oviduct fluid (SOF) medium supplemented with $8 \mathrm{mg} \mathrm{mL}^{-1}$ BSA-FAF, $0.2 \mathrm{mM}$ sodium pyruvate and $84.3 \mathrm{mg} \mathrm{mL}^{-1}$ amikacin in the absence of FBS in a controlled atmosphere of $5 \% \mathrm{CO}_{2}, 5 \% \mathrm{O}_{2}$ and $90 \% \mathrm{~N}_{2}$.

\section{Evaluation of nuclear maturation and cortical granule distribution}

We assessed nuclear maturation of oocytes based on meiotic progression to metaphase II (MII) and extrusion of the first polar body. Cytoplasmic maturation was inferred by cortical granule (CG) migration to the cortical region of the oocyte, following the methodology described by Cherr et al. (1988) with modifications. Briefly, we used $\sim 112$ oocytes per group for evaluation. For cytoplasmic maturation analyses, denuded oocytes, following the removal of the zona pellucida by $0.5 \%$ pronase in phosphate-buffered saline (PBS), were permeabilised with $0.1 \%$ Triton $\mathrm{X}-100$ for $5 \mathrm{~min}$ at $38^{\circ} \mathrm{C}$ and incubated with $10 \mu \mathrm{g} \mathrm{mL}^{-1}$ Lens culinaris agglutinin conjugated to fluorescein isothiocyanate (FITC-LCA) for $15 \mathrm{~min}$. For nuclear assessment, denuded oocytes were stained with $10 \mu \mathrm{g} \mathrm{mL}^{-1}$ Hoechst 33342 followed by PBS washes. To visualise the structures, we used an epifluorescence microscope (Olympus IX-FLA-70; Olympus, Tokyo, Japan) on the $40 \times$ objective, using filters with excitation of $330-385 \mathrm{~nm}$ and $420-490 \mathrm{~nm}$ emissions to view the nuclear stage and a filter with 460 $490 \mathrm{~nm}$ and $515 \mathrm{~nm}$ excitation and emission, respectively, for the cortical granules. Oocytes at MII with the presence of the first polar body were considered positive for nuclear maturation

P4

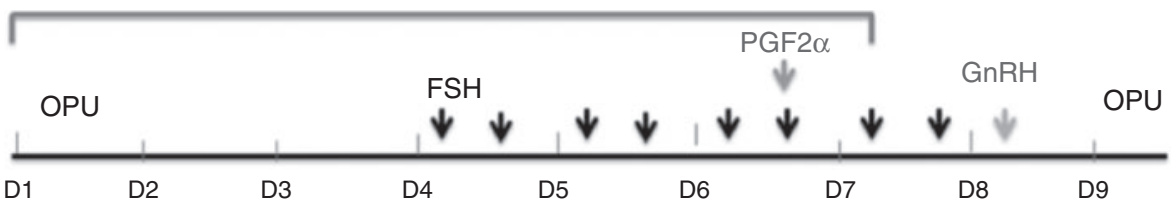

Fig. 1. Synchronisation and overstimulation protocol of in vivo-matured oocyte donors. Cows were synchronised after dominant follicle aspiration by OPU and overstimulated by administration of decreasing doses of FSH and a dose of GnRH $24 \mathrm{~h}$ before OPU for collection. 

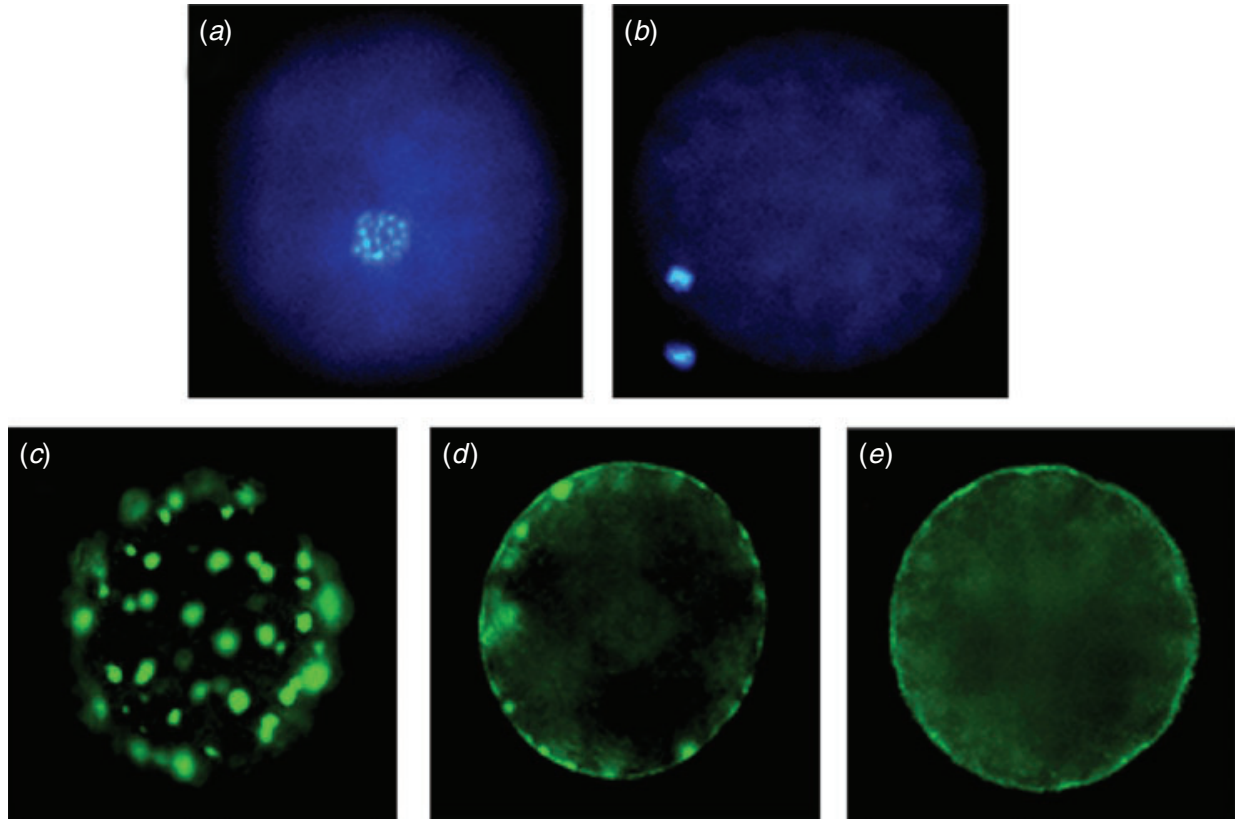

Fig. 2. Photomicrograph of nuclear progression and cortical granule distribution in bovine oocytes. Regarding the evaluation of nuclear progression, $(b)$ oocytes in MII and with the presence of the first polar body were considered mature, while $(a)$ those in other stages were considered immature. Regarding cortical granule migration, oocytes with cortical granules at $(e)$ the periphery forming a halo were considered mature and oocytes with the presence of $(c)$ 'clusters' or $(d)$ in transition were considered immature. Images were captured on $40 \times$ objective.

(Fig. 2b), while other nuclear stages were considered immature (Fig. 2a). For evaluation of cytoplasmic maturation, oocytes with cortical granules arranged at the periphery, forming a halo (Fig. 2e), were considered matured, whereas oocytes with the presence of 'clusters' (Fig. 2c) or in transition (Fig. 2d), were considered immature.

\section{Comparative analysis of lipid-droplet and mitochondrial behaviour during in vitro and in vivo maturation}

We evaluated immature and in vitro-matured oocytes as well as oocytes collected in vivo following the treatment for follicular overstimulation-maturation described above, for distribution and quantity of lipid droplets and mitochondria. For in vivomatured oocytes, collected by OPU, meiotic progression was evaluated as described above for in vitro-matured oocytes. We evaluated all oocytes ( $\sim 60$ per group) regarding the distribution and quantity of lipid droplets and mitochondria simultaneously. After being stripped, we first stained oocytes for visualisation of mitochondria with $0.5 \mathrm{mM}$ Mitotracker Red CMXRos (Molecular Probes, Eugene, OR, USA) for $30 \mathrm{~min}$ at $37^{\circ} \mathrm{C}$, which stains active mitochondria in living cells. Afterwards, oocytes were fixed in $4 \%$ formaldehyde for $30 \mathrm{~min}$. For analysis of lipid droplets, we permeabilised the fixed oocytes with $0.1 \%$ saponin for $30 \mathrm{~min}$ and stained with $5 \times$ HCS LipidTOX Green Neutral Lipid Stain (Molecular Probes) in $\mathrm{Ca}^{2+}$ - and $\mathrm{Mg}^{2+}$ - free PBS. Next, oocytes were analysed by confocal microscopy using a TCS-SP5 AOBS (Leica, Soims, Germany). We evaluated the distribution of structures in $\sim 60$ oocytes per group and captured images of 36 oocytes per group for quantification, obtained in five separate routines. For lipid-droplet distribution, we classified oocytes based on droplet location on the cytoplasm, as 'periphery' or 'dispersed' and, for mitochondria, we included a 'transition' category (Figs $3 a-e$ ). All images were captured under the same parameters, performing sequential acquisition. We used an argon laser for visualisation of lipid droplets and the excitation and emission were set to $488 \mathrm{~nm}$ and $500-537 \mathrm{~nm}$, respectively. For mitochondria, we used a $\mathrm{HeNe}$ laser with excitation and emission set at $543 \mathrm{~nm}$ and 580 $650 \mathrm{~nm}$, respectively. We captured three images of each oocyte: one in the middle of the oocyte (the image with largest diameter) and the other two in the middle of the resulting halves. We used a $40 \times$ objective at a resolution of $1024 \times 1024$ and images were analysed with the ImageJ program (NIH; http://rsb.info.nih.gov/ ij/). For mitochondrial activity, fluorescence intensity in the cytoplasm (Fig. $3 f$ ) was measured, averaging three sections per oocyte, with software assignment of intensity values between 0 and 255 for each pixel. For lipid quantification, we used the 'nucleus counter' tool, set to detect, distinguish and quantify droplet areas (Fig. 3g). The quantity of lipid in oocytes was obtained by analysis of the total area of lipids in each slide and obtaining the average of three sections ( $\mu \mathrm{m}^{2}$ of lipid per oocyte). In oocytes where all lipid droplets could be totally individualised (10 oocytes per group, 30 images per group), we also evaluated 

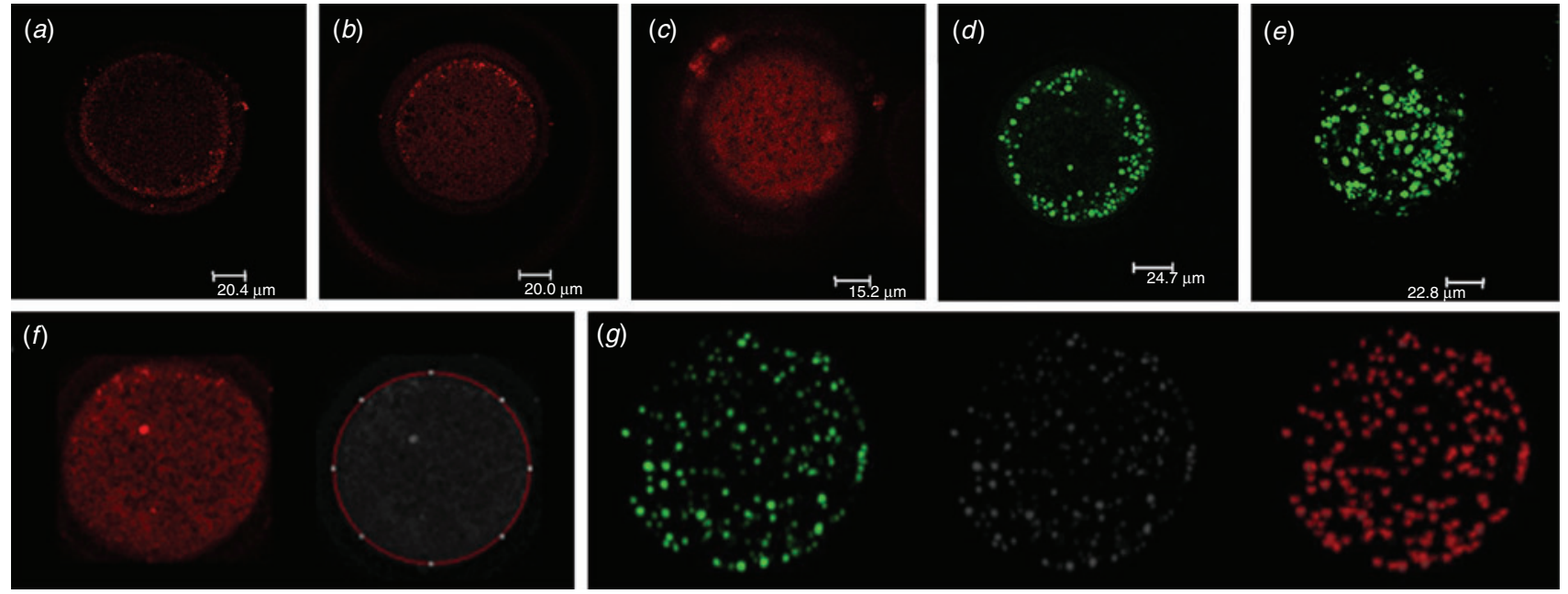

Fig. 3. Photomicrograph illustrating different categories of mitochondrial and lipid-droplet distribution in oocytes, and processing for mitochondrial and lipid quantification. Regarding mitochondrial distribution, they were characterised as $(a)$ periphery, $(b)$ transition, where most mitochondria were dispersed in the cytoplasm but with some accumulated in a minor part of the periphery or $(c)$ dispersed, where mitochondria were located homogeneously distributed throughout the cytoplasm. Regarding the distribution of lipid droplets, they were ranked as being $(d)$ in the periphery or $(e)$ scattered. ImageJ software was used to quantify the structures, which for $(f)$ mitochondria was performed measuring the fluorescence intensity after conversion of the image to 8 bits and delimitation of cytoplasm. $(g)$ For lipids the droplets were identified by the 'nucleus counter' tool of ImageJ. After conversion of the images to 8 bits, the tool located and individualised each droplet.

droplet sizes and classified into $<2 \mu \mathrm{m}, 2-6 \mu \mathrm{m}$ and $>6 \mu \mathrm{m}$ in diameter (Abe et al. 2002).

\section{Embryo development rates and lipid quantification}

We evaluated cleavage and blastocyst rates on the Day 2 and Day 8 of culture, respectively. The overall methodology for quantification and analysis of lipid-droplet size in embryos was performed as previously explained for oocytes, with minor modifications. For blastocysts, lipid quantity was corrected by area, to account for varying embryo sizes. After verification of a significant correlation $\left(\mathrm{r}^{2}=0.76\right.$ and $P<0.0001$ by Pearson's correlation test) between lipid quantity of three sections in 30 embryos ( 10 per group) we chose the section with the largest area per embryo to be analysed. We analysed 62,64 and 82 embryos for groups BSA, FBS and B $+F$, respectively. For the analysis of droplet size, we chose 15 sections of 15 embryos per group.

PLIN2 and DGAT2 expression in immature and in vitromatured oocytes and IVP embryos

For reverse transcription polymerase chain reaction (RT-PCR) we used cumulus cells from three pools of 50 immature COCs and COCs matured in different media (BSA, FBS and B+F), three pools from 90 denuded oocytes of each group (immature and matured in vitro) and three pools of 30 embryos from the three IVM groups. RNA extraction of samples was performed using the RNeasy Protect Micro kit (Qiagen, Valencia, CA, USA) according to the manufacturer's instructions and reverse transcription was performed using the oligodT primer and Superscript III kit (Invitrogen, Carlsbad, CA, USA). Using the Quantitect SyberGreen kit (Qiagen) according to the manufacturer's recommendations, we performed real-time PCR reactions in a Mx3005P QPCR System from Stratagene (La Jolla, CA, USA). PCR reactions were conducted in triplicate and expression was determined based on the ratio of each gene over the control, glyceraldehyde-3-phosphate dehydrogenase $(G A P D H)$. A gene-specific standard curve was utilised at each run for quantification. PLIN2 and DGAT2 primers were designed, based on the bovine sequences available on the genome browser of University of California Santa Cruz (UCSC) (http:// genome.ucsc.edu, accessed December 2013), using the program Primer3 (Primer3web, version 4.0.0: http://primer3.ut.ee), and synthesised by Sigma Aldrich. The GAPDH primers, used as endogenous control gene, were obtained from the company Promidol (Alere, Belo Horizonte, Brazil). The primer sequences were: PLIN2 (product size: $149 \mathrm{bp}$ ) F $5^{\prime}$ TGCACTCACC AAATCAGAGC 3', R 5' GCAGCTTGGTGGACAGAGAT 3'; DGAT2 (product size: $152 \mathrm{bp}$ ): F $5^{\prime}$ TTGGCTCAATAGG TCCAAGG $3^{\prime}, \mathrm{R} 5^{\prime}$ TGAAGTAGAGCACGGCAATG 3'; GAPDH (NM_001034034; product size: 76bp) F 5' AAGG CCATCACCATCTTCCA $3^{\prime}, \mathrm{R} 5^{\prime}$ CCACTACATACTCAGCA CCAGCAT $3^{\prime}$.

\section{Statistical analysis}

We evaluated nuclear maturation and cortical-granule migration rates, as well as embryo development rates by chi-square $(\chi 2)$ test or, when appropriate, the Fisher exact test. Lipid quantification in oocytes and embryos, as well as mitochondrial activity quantification, were assessed by non-parametric Mann-Whitney test. The correlation between the structures in the oocytes was determined by linear regression $\mathrm{r} 2$ test and significance of the correlation by $\mathrm{F}$ test. For gene-expression 
evaluation, we used analysis of variance (ANOVA) and Duncan's multiple-range test. The results were analysed with the GraphPad Instat 1.6 program (GraphPad Software, Inc., La Jolla, CA, USA), with the exception of gene-expression data, which were evaluated using the XLSTAT (2012) program (XLSTAT, New York, NY, USA).

\section{Results}

\section{FBS lipid content}

Aliquots of FBS were subjected to chromatography for the separation of fatty acids (Fig. $4 a$ ). The graph generated allowed for FBS lipid content determination $(0.029 \mathrm{~g}$ per $100 \mathrm{~mL})$ and fatty-acid profiling. Based on this result, we estimated lipid quantity for each IVM medium (Fig. 4b), revealing that medium containing FBS has eighteen times more fatty acids $\left(0.029 \mathrm{mg} \mathrm{mL}^{-1}\right)$ than medium containing BSA only

(a)

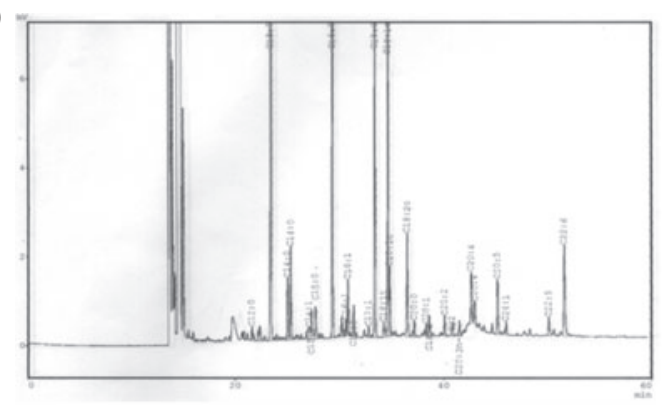

(b)

\begin{tabular}{cccc}
\hline Groups & BSA & FBS & B $+F$ \\
& & & \\
\hline $\mathrm{mg}$ of lipid/mL & 0.0016 & 0.029 & 0.0157 \\
& & & \\
\hline
\end{tabular}

(c)

\begin{tabular}{ccc}
\hline Name & $\mathrm{mg} / 100 \mathrm{~mL}$ & $\%$ area \\
\hline Saturated & $14.12 \pm 2.23$ & $47.89 \pm 4.89$ \\
Monounsaturated & $7.3 \pm 0.79$ & $22.4 \pm 1.58$ \\
Polyunsaturated & $4.99 \pm 1.67$ & $16.57 \pm 3.48$ \\
Trans & 0.46 & $1.50 \pm 0.73$ \\
Not identified & & $11.64 \pm 3.11$ \\
\hline
\end{tabular}

$\left(0.0016 \mathrm{mg} \mathrm{mL}^{-1}\right)$ and nearly twice as much as medium using the combination of the two protein sources, B+F $(0.0157 \mathrm{mg}$ $\left.\mathrm{mL}^{-1}\right)$. Of the fatty acids present in FBS, almost half $(47.89 \%)$ are saturated fatty acids (Fig. $4 c$ ), with palmitic acid making up $23.4 \%$ of the total, followed by stearic acid $(17.71 \%)$ and by the unsaturated oleic acid (15.24\%); Fig. $4 d$.

Presence of FBS during IVM results in higher rates of nuclear maturation and migration of cortical granules (CG)

Routinely, the rates of meiotic progression and migration of cortical granules are used as indicators of oocyte maturation. In the present work, we demonstrated the limitations of BSA-FAF in this process. BSA had lower rates of oocytes in MII with first polar body extrusion and oocytes with cortical granules in the periphery, compared with groups containing FBS (FBS and $\mathrm{B}+\mathrm{F})($ Table 1). (d)

\begin{tabular}{|c|c|c|c|}
\hline \multirow[t]{2}{*}{ Formula } & \multirow[t]{2}{*}{ Name } & \multicolumn{2}{|c|}{ FBS } \\
\hline & & $\mathrm{mg} / 100 \mathrm{~mL}$ & \%Area \\
\hline $12: 0$ & Lauric & $0.11 \pm 0.07$ & $0.32 \pm 0.28$ \\
\hline $14: 0$ & Myristic & $0.68 \pm 0.35$ & $2.59 \pm 1.02$ \\
\hline $15: 0$ & Pentadecanoic & $0.52 \pm 0.46$ & $1.07 \pm 0.52$ \\
\hline $16: 0$ & Palmitic & $6.82 \pm 1.08$ & $23.40 \pm 1.74$ \\
\hline $16: 1(n-7)$ & Palmitoleic & $0.64 \pm 0.14$ & $2.27 \pm 0.22$ \\
\hline $17: 0$ & Margaric & $0.42 \pm 0.05$ & $1.43 \pm 0.07$ \\
\hline $17: 1(n-7)$ & Heptadecenoic & $0.24 \pm 0.03$ & $0.72 \pm 0.18$ \\
\hline $18: 0$ & Estearic & $5.16 \pm 0.77$ & $17.71 \pm 3.51$ \\
\hline $18: 19 t$ & Elaidic & $0.46 \pm 0.18$ & $1.50 \pm 0.73$ \\
\hline $18: 1(n-9)$ & Oleic & $5.01 \pm 0.74$ & $15.24 \pm 1.01$ \\
\hline $18: 1(n-7)$ & Vaccenic & $1.09 \pm 0.05$ & $3.51 \pm 0.47$ \\
\hline $18: 2(n-6)$ & Linoleic & $1.51 \pm 0.48$ & $5.13 \pm 1.12$ \\
\hline $20: 0$ & Eicosanoic & $0.14 \pm 0.07$ & $0.49 \pm 0.17$ \\
\hline $20: 1(n-9)$ & Eicosenoic & - & - \\
\hline $18: 3(n-3)$ & $\alpha$-Linolenic & $0.17 \pm 0.16$ & $0.67 \pm 0.42$ \\
\hline $22: 0$ & Docosanoic & $0.28 \pm 0.05$ & $0.88 \pm 0.17$ \\
\hline $22: 1$ & Docosenoic & - & - \\
\hline $20: 3(n-6)$ & Eicotrienoic & $0.40 \pm 0.08$ & $1.24 \pm 0.41$ \\
\hline $20: 4(n-6)$ & Arachidonic & $1.00 \pm 0.09$ & $3.10 \pm 0.56$ \\
\hline $24: 0$ & Lignoceric & - & - \\
\hline $20: 5(n-3)$ & Eicosapentaenoic & $0.42 \pm 0.40$ & $1.56 \pm 1.17$ \\
\hline $24: 1$ & Nervonic & $0.33 \pm 0.12$ & $0.65 \pm 0.38$ \\
\hline $22: 5(n-3)$ & Docosapentaenoic & $0.50 \pm 0.08$ & $1.45 \pm 0.31$ \\
\hline $22: 6(n-3)$ & Docosahexaenoic & $0.99 \pm 0.81$ & $3.42 \pm 2.37$ \\
\hline
\end{tabular}

Fig. 4. FBS gas chromatography. (a) Graphic illustration of chromatography results, where each peak refers to each fattyacid area and is used to determine the acid ratio in the sample. (b) Table with lipid quantity in each IVM medium. $(c, d)$ Tables with each fatty-acid proportion in the sample. 
IVM affects distribution and quantity of lipids and active mitochondria

Following the observation of a high lipid content in FBS and its significance on nuclear maturation and the migration of cortical

Table 1. Rates of bovine oocytes in MII with cortical granules (CG) arranged peripherally after $24 \mathrm{~h}$ of in vitro maturation with the different supplements

BSA, $8 \mathrm{mg} \mathrm{mL}^{-1}$ BSA-FAF; FBS, $10 \%$ FBS; B +F, $6 \mathrm{mg} \mathrm{mL}^{-1}$ BSA-FAF + $5 \%$ FBS. ${ }^{\text {a,b }}$ Values in the same column with different superscripts differ by $\chi 2$ test $(P<0.05)$

\begin{tabular}{lcc}
\hline Group & Oocytes in MII $n(\%)$ & Oocytes with CG in periphery $n(\%)$ \\
\hline BSA & $69 / 92(75.0)^{\mathrm{a}}$ & $54 / 123(43.9)^{\mathrm{a}}$ \\
FBS & $104 / 117(88.9)^{\mathrm{b}}$ & $78 / 125(62.4)^{\mathrm{b}}$ \\
$\mathrm{B}+\mathrm{F}$ & $95 / 106(89.6)^{\mathrm{b}}$ & $58 / 113(51.3)^{\mathrm{a}, \mathrm{b}}$ \\
\hline
\end{tabular}
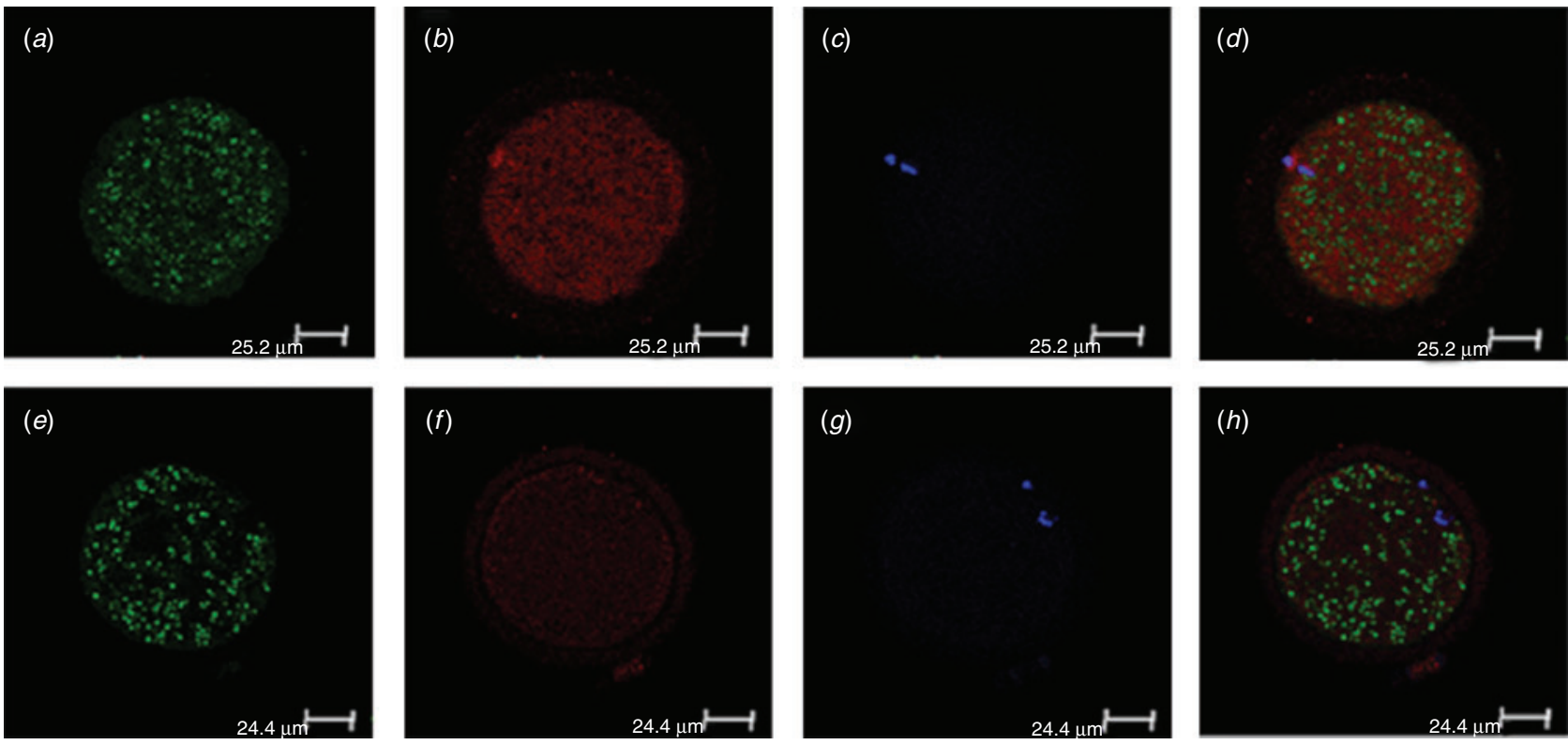

Fig. 5. Lipid and mitochondrial behaviour during in vivo maturation in MII oocytes; examples of asynchronous and synchronous migration of structures in in vivo MII oocytes. Locations of $(a, e)$ lipid droplets, $(b, f)$ mitochondria and $(c, g)$ nucleus at MII. $(d, h)$ Merged images. $(a-d)$ Oocyte showing synchronous migration of lipid droplets and mitochondria. $(e-h)$ Oocyte representing asynchronous migration of these structures.

Table 2. Rates of oocytes in different categories for the location of mitochondria (peripheral, transition and dispersed) and lipid droplets (periphery and dispersed) in immature, in vitro-matured (BSA, FBS and $\mathrm{B}+\mathrm{F}$ ) and in vivo-matured oocytes

BSA, $8 \mathrm{mg} \mathrm{mL}^{-1}$ BSA-FAF; FBS, $10 \%$ FBS; B $+F, 6 \mathrm{mg} \mathrm{mL}^{-1}$ BSA-FAF $+5 \%$ FBS. ${ }^{\text {a,b,c }}$ Values in the same column with different superscripts differ by $\chi^{2}$ test $(P<0.05)$

\begin{tabular}{|c|c|c|c|c|c|}
\hline \multirow[t]{2}{*}{ Group } & \multicolumn{3}{|c|}{ Mitochondria } & \multicolumn{2}{|c|}{ Lipid droplets } \\
\hline & Periphery $n(\%)$ & Transition $n(\%)$ & Dispersed $n(\%)$ & Periphery $n(\%)$ & Dispersed $n(\%)$ \\
\hline Immature & $55 / 59(93.2)^{\mathrm{a}}$ & $0 / 59^{a}$ & $4 / 59(6.8)^{\mathrm{a}}$ & $36 / 59(61)^{\mathrm{a}}$ & $23 / 59(39)^{a}$ \\
\hline BSA & $42 / 61(68.8)^{b}$ & $12 / 61(19.7)^{b, c}$ & $7 / 61(11.5)^{\mathrm{a}}$ & $17 / 61(27.9)^{\mathrm{b}}$ & $44 / 61(72.1)^{b}$ \\
\hline FBS & $25 / 65(38.5)^{\mathrm{c}}$ & $19 / 65(29.23)^{b}$ & $21 / 65(32.3)^{b}$ & $2 / 65(3.1)^{c}$ & $63 / 65(96.9)^{\mathrm{c}}$ \\
\hline $\mathrm{B}+\mathrm{F}$ & $33 / 66(50)^{\mathrm{b}}$ & $23 / 66(34.8)^{b}$ & $10 / 66(15.1)^{\mathrm{a}}$ & $3 / 66(4.5)^{\mathrm{c}}$ & $63 / 66(95.4)^{\mathrm{c}}$ \\
\hline In vivo & $22 / 58(37.9)^{\mathrm{c}}$ & $6 / 58(10.3)^{c}$ & $30 / 58(51.7)^{\mathrm{c}}$ & $12 / 58(20.7)^{b}$ & $46 / 58(79.3)^{\mathrm{b}}$ \\
\hline
\end{tabular}


of the IVM groups achieved similar migration rates to those seen in vivo, the rates observed for the FBS group were the closest to those in vivo (Table 2).

When we analysed lipid and active mitochondria values of immature, in vivo- and in vitro-matured oocytes, we noticed higher levels of lipid accumulation during IVM in comparison to those oocytes that underwent hormonal stimulation in the in vivo group. Furthermore, the FBS group had higher accumulation compared with the group supplemented only with BSA (Fig. 6a). Supplementation during IVM did not affect oocyte lipid-droplet sizes (Fig. 6b). However, as illustrated in Fig. $6 c$, the only group able to increase mitochondrial activity during maturation was the BSA group, showing significantly higher values than those observed in the groups containing serum (FBS and $\mathrm{B}+\mathrm{F})$. Interestingly, we observed a decrease in mitochondrial activity in the in vivo group (Fig. 6c). Notwithstanding, we analysed the correlation between active mitochondria and lipid droplets within each group, and the only group able to maintain a significant correlation between the structures was the in vivo group (Fig. 6d).

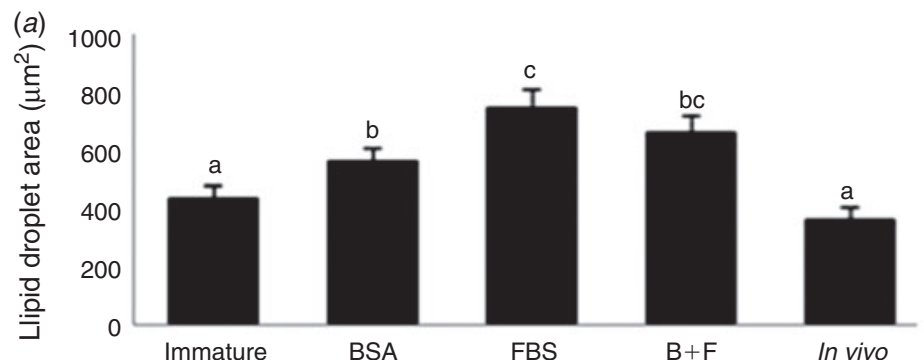

(c)

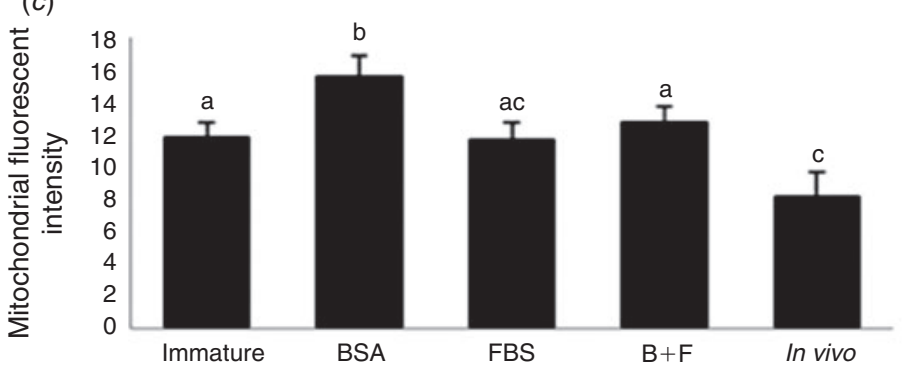

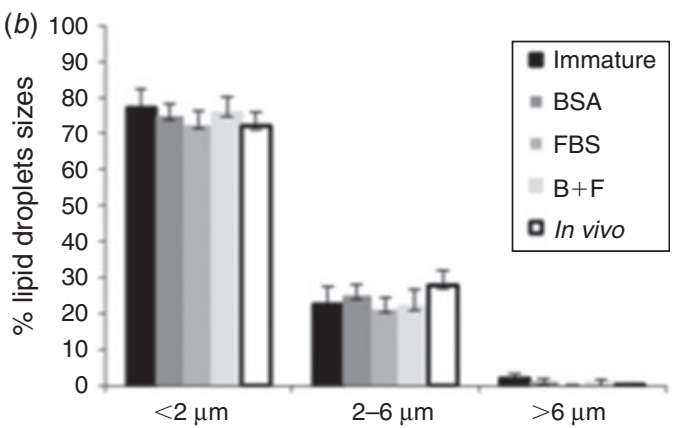
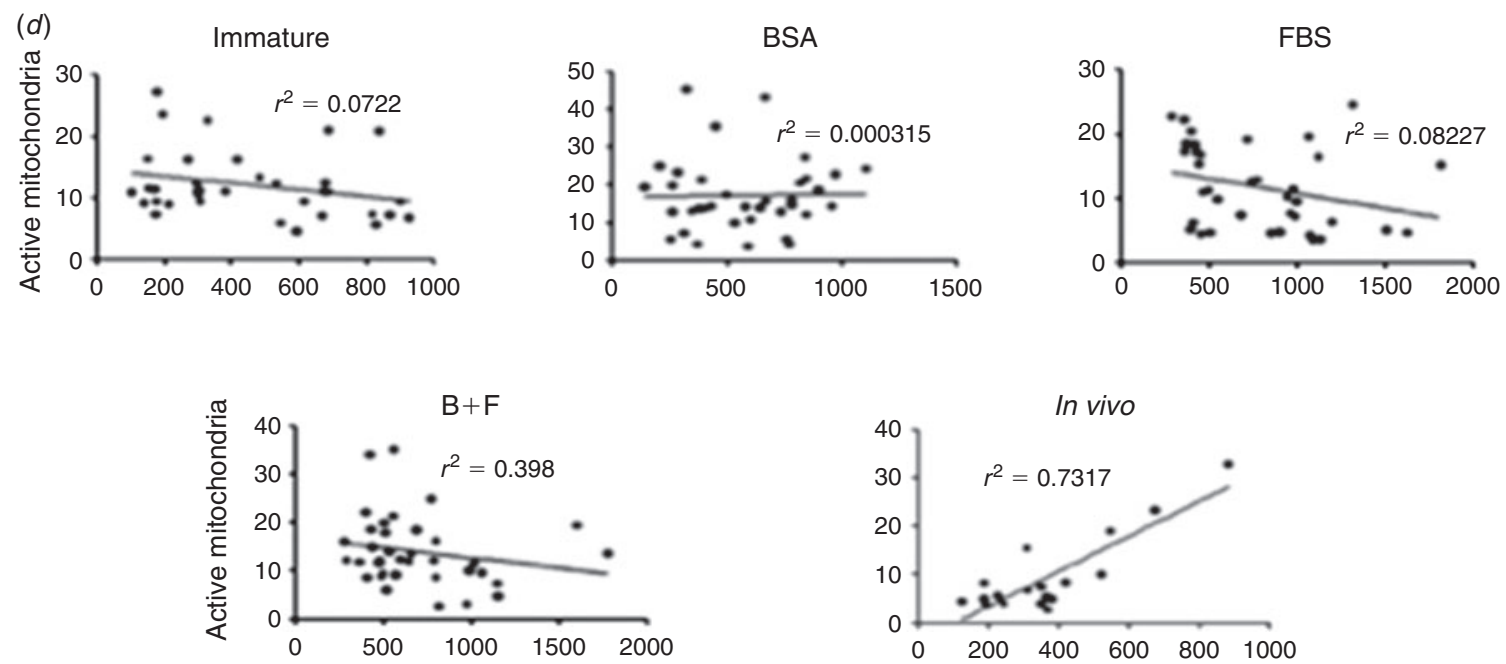

Lipids

Fig. 6. Behaviour and quantity of lipid droplets and active mitochondria during IVM and in vivo maturation. $(a, c)$ Amount of lipid and numbers of active mitochondria in immature $(n=41)$, BSA $(n=39)$, FBS $(n=41), \mathrm{B}+\mathrm{F}(n=40)$ and in vivo-matured $(n=20)$ oocytes. (b) Lipid-droplet size classification of oocyte groups. (d) Correlation between mitochondria and lipid droplets in the different groups. 
Reduction in FBS concentration during IVM lowers the amount of lipid in embryos

BSA supplementation during IVM resulted in a significant reduction in the rate of blastocyst formation after IVF and IVC (Table 3). Interestingly, the $\mathrm{B}+\mathrm{F}$ group, containing half the concentration of FBS used in standard medium, allowed for a

Table 3. Cleavage and blastocyst rates after IVM of oocytes in different media (BSA, FBS and B+F), fertilisation and in vitro culture for 8 days in the absence of FBS

BSA, $8 \mathrm{mg} \mathrm{mL}^{-1}$ BSA-FAF; FBS, $10 \%$ FBS; B +F, $6 \mathrm{mg} \mathrm{mL}^{-1}$ BSA-FAF + $5 \%$ FBS. ${ }^{\mathrm{a}, \mathrm{b}}$ Values in the same column with different superscripts differ by $\chi 2$ test $(P<0.05)$

\begin{tabular}{lrrc}
\hline Group & \multicolumn{1}{c}{ Cleaved $n(\%)$} & Blastocysts $n(\%)$ & Blastocysts/cleaved $n(\%)$ \\
\hline BSA & $1232 / 1410(87.38)^{\mathrm{a}}$ & $279 / 1410(19.79)^{\mathrm{a}}$ & $279 / 1232(22.65)^{\mathrm{a}}$ \\
FBS & $844 / 927(91.05)^{\mathrm{b}}$ & $336 / 927(36.25)^{\mathrm{b}}$ & $336 / 844(39.81)^{\mathrm{b}}$ \\
B+F & $959 / 1062(90.30)^{\mathrm{b}}$ & $368 / 1062(34.65)^{\mathrm{b}}$ & $368 / 959(38.37)^{\mathrm{b}}$ \\
\hline
\end{tabular}

reduction in total lipid amount in the embryos, while maintaining embryo production rates similar to those obtained by FBS alone (Fig. 7a). IVM supplementation did not influence the size of the lipid droplets present in the embryos (Fig. 7b).

\section{IVM causes changes in PLIN2 and DGAT2 expression in oocytes, but not in embryos}

In order to understand how supplementation during IVM influences lipid metabolism of oocytes and embryos, we investigated the expression of PLIN2 and DAGT2 in cumulus cells, immature and in vitro mature denuded oocytes and in IVM-derived embryos (Fig. 8). We observed different patterns of expression for DGAT2 and PLIN2 in cumulus cells. Regardless of the treatment, PLIN2 expression was increased in cumulus cells from matured oocytes, whereas DGAT2 showed decreased expression in these cells following IVM. Among the denuded oocytes, the only significant difference found was in the FBS group, which showed a decreased expression of DGAT2 compared with the immature group. Treatments during IVM did not affect DGAT2 and PLIN2 expression in blastocysts.
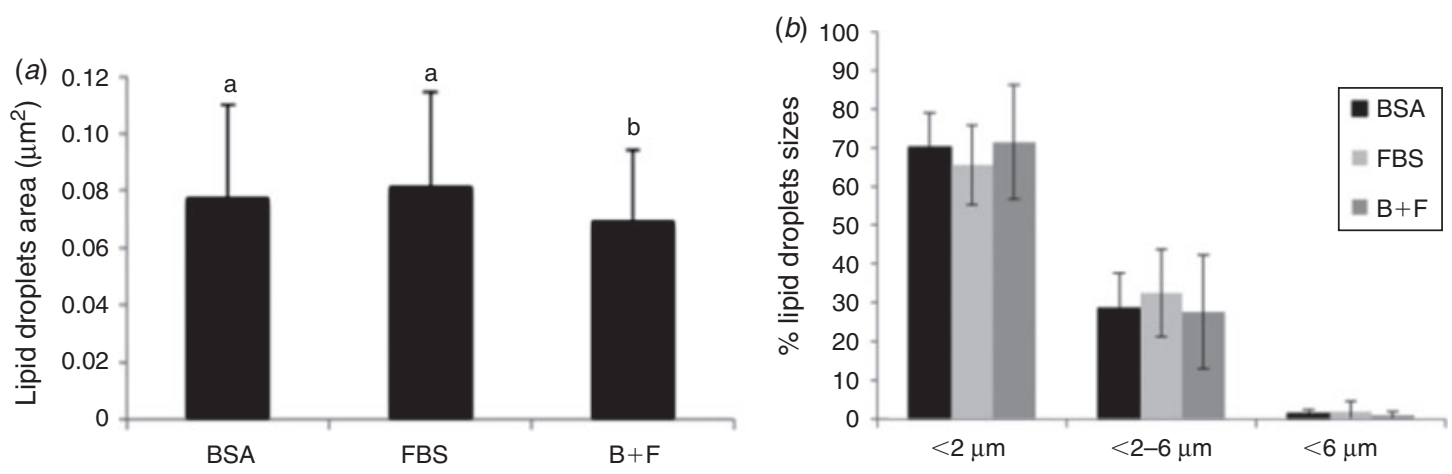

Fig. 7. Embryo lipid analysis. (a) Amount of lipid in blastocysts of the IVM groups: BSA, FBS and B+F. (b) Lipid-droplet size evaluation in embryos following IVM.

(a)

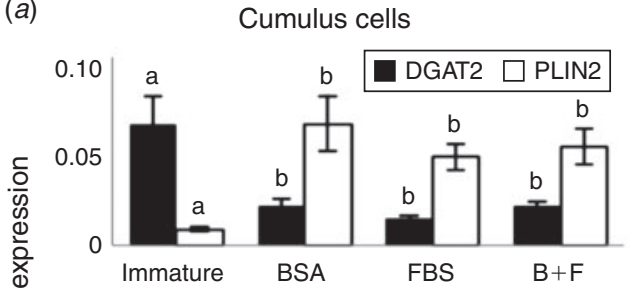

(b)

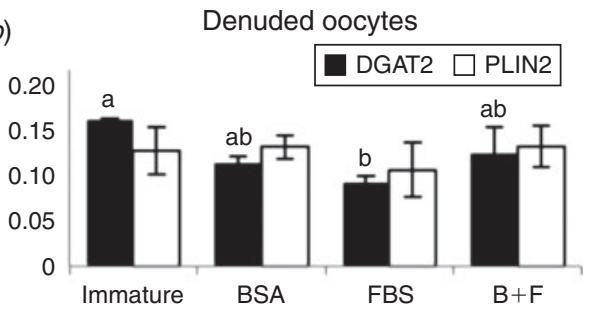

$(c)$

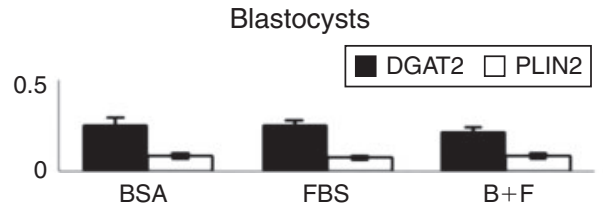

Fig. 8. Expression of DGAT2 and PLIN2 in (a) cumulus cells, (b) IVM oocytes (BSA, FBS and B+F) and (c) subsequent blastocysts. Different letters for the same gene indicate significant differences $(P<0.05)$. 


\section{Discussion}

To our knowledge, the present study is the first to determine the total lipid content of FBS. We confirmed that serum has a much higher concentration of fatty acids than the other commonly used media supplement, BSA-FAF. These findings support the hypothesis that the increased lipid accumulation found in oocytes matured in the presence of FBS may be, in part, due to lipid transport from the medium to the cytoplasm of the oocyte. In fact, saturated fatty acids, prevalent in FBS, can penetrate the oocyte (Adamiak et al. 2006). The elevated concentration of palmitic acid found in FBS also concurs with this idea, as previous researchers have shown that oocytes and embryos, when cultured in the presence of FBS, have a high concentration of this particular fatty acid (Sata et al. 1999; Kim et al. 2001; Ferreira et al. 2012). In the present study, oleic acid is the third most-abundant in FBS, and it is known that this fatty acid is predominant in the follicular fluid (Tsujii et al. 2001; Leroy et al. 2005). It is possible that this change in the ratio of palmitic/oleic acid adversely affects IVP. Harmful effects of excess palmitic acid in both maturation and embryo development (Leroy et al. 2005), and positive effects of oleic acid in the same stages of development (Aardema et al. 2011), have been reported. We consider that the difference in lipid profile between follicular fluid and FBS, normally used in IVM and in vitro culture, could be one of the causes for the observed differences and outcomes between in vitro and in vivo embryos in cattle.

Despite possible deleterious effects of FBS, we suggest that elements present in this supplement promote, in addition to nuclear maturation, the migration of organelles during IVM. We found that $5 \% \mathrm{FBS}$, half of the concentration commonly used in maturation media, resulted in satisfactory migration of cortical granules and meiotic progression (Table 1). On the other hand, the supplementation with BSA only during IVM prevented adequate nuclear maturation and CG migration rates. A decrease in nuclear maturation in oocytes cultured with BSA-FAF during IVM has been described elsewhere (Ali and Sirard 2002). This result can be explained by the effects that fatty acids, and their oxidation, have on meiotic progression (Downs et al. 2009). One such effect is demonstrated by the activation of mitogenactivated protein kinase (MAPK) eliciting germinal-vesicle breakdown and reactivation of meiosis (Chen et al. 2006). According to Downs et al. (2009), MAPK phosphorylates acetyl-CoA carboxylase (ACC), thus deactivating it and decreasing cellular malonyl-CoA levels, which is an oxidative pathway inhibitor. Also, the relationship between $\beta$-oxidation and nuclear maturation rates were shown in cattle, pigs and mice (Downs et al. 2009; Dunning et al. 2010; Paczkowski et al. 2013).

Dunning et al. (2010) showed the importance of oocyte oxidation for embryo development and found that, although there is increased oxidation during IVM in mice, maturation in the presence of FBS results in an even higher oxidation rate, with positive consequences in embryo development when compared with IVM supplemented with BSA only. The authors refer to a possible lack of L-carnitine from medium composed solely of BSA, as L-carnitine is responsible for transporting fatty acids into the mitochondrial membrane for $\beta$-oxidation to occur (Downs et al. 2009; Dunning et al. 2010). BSA-FAF is substantially free of fatty acids. This fact, associated with a possible limitation in the oxidation described above for murine oocytes matured in this supplement, could be a plausible explanation for the lower meiotic progression rate we have observed in oocytes matured with this supplement as the sole protein source, during the present study.

Although the rates of mitochondrial migration in the in vivo group were not as high as previously reported in the literature (Hyttel et al. 1986), mitochondrial migration appears to be negatively affected by the in vitro system. None of the IVM treatment groups were able to reach the values observed in oocytes matured in vivo, albeit FBS provided the highest rate of migration of the three IVM groups. However, despite lower rates of mitochondrial migration, the group that utilised intermediate concentrations of the two supplements showed similar rates of maturation and blastocyst formation, arguing that incomplete mitochondrial migration does not appear to affect oocyte competence and subsequent embryonic development.

The fact that lipid accumulation was present in oocytes of all three groups following IVM but was not observed in the in vivo group, suggests that, in addition to protein supplementation, there are other factors in the in vitro system affecting lipid metabolism. It is possible that during physiological maturation (in vivo), lipid synthesis occurs concomitantly with its metabolism by oxidation, impeding excessive lipid accumulation. This hypothesis is supported by the high correlation between lipids and mitochondria observed for the in vivo group. Lipid accumulation following IVM, shown in the present study, is in accordance with current literature reports (Ferreira et al. 2008; Aardema et al. 2011; González-Serrano et al. 2013). In the same way, an increase in lipid quantity during IVM was observed when culture media contained only BSA (Aardema et al. 2011). Moreover, reports have demonstrated an increase in the expression of genes and proteins of lipid metabolism during IVM in lipid-free medium (Auclair et al. 2013; Dunning et al. 2014). Those data suggest an increase in lipid accumulation not only by lipid incorporation, but also by affecting lipid metabolism during IVM.

Unlike the oocytes recovered after animal hormonal stimulation, oocytes undergoing IVM with higher concentrations of FBS failed to maintain correlation between active mitochondria and lipid content. Furthermore, the increase in lipid accumulation in this group was not accompanied by an increase in active mitochondria, which may have influenced the efficiency of oxidation of these lipids. Regarding oocytes matured in the presence of BSA-FAF, although the group was able to increase the amount of active mitochondria and lipids, the physical distance between the structures, caused by asynchrony during migration, along with a previously suggested lack of L-carnitine and consequently low levels of oxidation (Dunning et al. 2010), could have caused the low rates of maturation and embryo development observed in this group. All of these differences may have affected the ability to metabolise lipids accumulated during IVM in this experimental group, and possibly further during IVC, as no differences in lipids were observed in blastocysts from the BSA and FBS groups. As suggested by Dunning et al. (2010) the group containing 10\% FBS could 
have promoted higher $\beta$-oxidation rates. However, serum has detrimental effects on mitochondrial membranes (Russell et al. 2006), which, associated with the high lipid concentration in this supplement, may have prevented the metabolism of such large amounts of fatty acids, worsened by the high lipid intake by the embryos. In contrast, the combination of BSA-FAF with 5\% FBS, analysed in this study, possibly allowed for a better balance between intake and oxidation, while providing acceptable maturation and embryo development rates and lower lipid accumulation in embryos.

Despite its effects in lipid accumulation on oocytes and embryos, IVM supplementation had no effect on lipid-droplet sizes during our study, in agreement with a previous literature report (Aardema et al. 2011).

Expression of PLIN2 and DGAT2 in cumulus cells during IVM suggests an increase in lipid synthesis and this could be related to the lipid accumulation observed in the oocyte cytoplasm. This lipid accumulation in cumulus cells likely happens to provide energy to the cells, to supply the needs of these steroidogenic cells or perform the transportation of lipids to the oocyte. For PLIN2, an increase in mRNA could be justified by increased demand for lipid-droplet formation, and for DGAT2, the decrease in mRNA may be linked to increased protein translation and production of this enzyme required for lipid synthesis. However, despite the differences observed in embryo lipid accumulation between groups, supplementation during IVM did not affect these genes involved in lipid metabolism in embryos.

In conclusion, we noted improper migration of mitochondria and lipid droplets following IVM, and the possible effects that could carry over through the in vitro process, affecting not only development rates but also lipid accumulation. Furthermore, we have shown that lowering the FBS concentration to 5\% may be an interesting alternative, since it enabled the reduction of lipid accumulation in embryos without adversely affecting oocyte maturation and embryo development.

\section{Acknowledgements}

We are grateful to the staff of the Animal Reproduction Laboratory of the Departamento de Medicina Veterinária Preventiva e Reprodução Animal (UNESP, Jaboticabal, Brazil) for their support during this work. We thank Prof. Felipe Perecin (FZEA (Faculdade de Zootecnia e Engenharia de Alimentos- FZEA, USP, Pirassununga, Brazil), USP, Brazil) for his help with the statistical analysis. We also would like to thank Dr Daniel Robert Arnold for proofreading the manuscript for English content. Supported by Coordenação de Aperfeiçoamento de Pessoal de Nível Superior (CAPES).

\section{References}

Aardema, H., Vos, P. L., Lolicato, F., Roelen, B. A., Knijn, H. M., Vaandrager, A. B., Helms, J. B., and Gadella, B. M. (2011). Oleic acid prevents detrimental effects of saturated fatty acids on bovine oocyte developmental competence. Biol. Reprod. 85(1), 62-69. doi:10.1095/ BIOLREPROD.110.088815

Abd El Razek, I. M., Charpigny, G., Kodja, S., Marquant-Leguienne, B., Mermillod, P., Guyader-Joly, C., and Humblot, P. (2000). Difference in lipid composition between in vivo- and in vitro-produced bovine embryos. Theriogenology 54(1), 346.

Abe, H., Yamashita, S., Satoh, T., and Hoshi, H. (2002). Accumulation of cytoplasmic lipid droplets in bovine embryos and cryotolerance of embryos developed in different culture systems using serum-free or serum-containing media. Mol. Reprod. Dev. 61(1), 57-66. doi:10.1002/ MRD. 1131

Adamiak, S. J., Powell, K., Rooke, J. A., Webb, R., and Sinclair, K. D. (2006). Body composition, dietary carbohydrates and fatty acids determine post-fertilisation development of bovine oocytes in vitro. Reproduction 131(2), 247-258. doi:10.1530/REP.1.00871

Ali, A., and Sirard, M. A. (2002). Effect of the absence or presence of various protein supplements on further development of bovine oocytes during in vitro maturation. Biol. Reprod. 66(4), 901-905. doi:10.1095/ BIOLREPROD66.4.901

Auclair, S., Uzbekov, R., Elis, S., Sanchez, L., Kireev, I., Lardic, L., Dalbies-Tran, R., and Uzbekova, S. (2013). Absence of cumulus cells during in vitro maturation affects lipid metabolism in bovine oocytes. Am. J. Physiol. Endocrinol. Metab. 304(6), E599-E613. doi:10.1152 AJPENDO.00469.2012

Bickel, P. E., Tansey, J. T., and Welte, M. A. (2009). PAT proteins, an ancient family of lipid-droplet proteins that regulate cellular lipid stores. Biochim. Biophys. Acta 1791(6), 419-440. doi:10.1016/J.BBALIP. 2009.04.002

Chen, J., Hudson, E., Chi, M. M., Chang, A. S., Moley, K. H., Hardie, D. G., and Downs, S. M. (2006). AMPK regulation of mouse oocyte meiotic resumption in vitro. Dev. Biol. 291(2), 227-238. doi:10.1016/J.YDBIO. 2005.11.039

Cherr, G. N., Drobnis, E. Z., and Katz, D. F. (1988). Localisation of cortical granule constituents before and after exocytosis in the hamster egg. J. Exp. Zool. 246(1), 81-93. doi:10.1002/JEZ.1402460111

Cummins, J. M. (2004). The role of mitochondria in the establishment of oocyte functional competence. Eur. J. Obstet. Gynecol. Reprod. Biol. 115(Suppl 1), S23-S29. doi:10.1016/J.EJOGRB.2004.01.011

Downs, S. M., Mosey, J. L., and Klinger, J. (2009). Fatty-acid oxidation and meiotic resumption in mouse oocytes. Mol. Reprod. Dev. 76(9), 844-853. doi:10.1002/MRD.21047

Dunning, K. R., Cashman, K., Russell, D. L., Thompson, J. G., Norman, R. J., and Robker, R. L. (2010). Beta-oxidation is essential for mouse oocyte developmental competence and early embryo development. Biol. Reprod. 83(6), 909-918. doi:10.1095/BIOLREPROD.110.084145

Dunning, K. R., Anastasi, M. R., Zhang, V. J., Russell, D. L., and Robker, R. L. (2014). Regulation of fatty-acid oxidation in mouse cumulus-oocyte complexes during maturation and modulation by PPAR agonists. PLOS One 9(2), e87327. doi:10.1371/JOURNAL.PONE.0087327

Ferguson, E. M., and Leese, H. J. (1999). Triglyceride content of bovine oocytes and early embryos. J. Reprod. Fertil. 116(2), 373-378. doi:10.1530/JRF.0.1160373

Ferreira, C. R., Catharino, R. R., Saraiva, S. A., Fonsêca, M. F. R., Basso, A. C., Pontes, J. H. F., Ereno, J. C., Jr, and Eberlin, M. N. (2008). Perspectivas para aplicação da técnica de espectrometria de massas na caracterização do lipidoma de oócitos e embriões bovinos produzidos in vitro. Acta Clientage Veterinarian 36(2), 549.

Ferreira, C. R., Eberlin, L. S., Hallett, J. E., and Cooks, R. G. (2012). Single oocyte and single embryo lipid analysis by desorption electrospray ionisation mass spectrometry. J. Mass Spectrom. 47(1), 29-33. doi:10.1002/JMS.2022

Gilbert, S. F. (2003). 'Developmental Biology, 7th edn.' (Sinauer Associates: Sunderland, MA.)

González-Serrano, A. F., Pirro, V., Ferreira, C. R., Oliveri, P., Eberlin, L. S., Heinzmann, J., Lucas-Hahn, A., Niemann, H., and Cooks, R. G. (2013) Desorption electrospray ionisation mass spectrometry reveals lipid metabolism of individual oocytes and embryos. PLoS One 8(9), e74981. doi:10.1371/JOURNAL.PONE.0074981

Hyttel, P., Callesen, H., and Greve, T. (1986). Ultrastructural features of preovulatory oocyte maturation in superovulated cattle. J. Reprod. Fertil. 76(2), 645-656. doi:10.1530/JRF.0.0760645 
IETS (2012). 'IETS 2012 Statistics and Data Retrieval Committee Report'. Available at http://www.iets.org/pdf/comm_data/December2012.pdf [Verified 24 April 2015]

Kim, J. Y., Kinoshita, M., Ohnishi, M., and Fukui, Y. (2001). Lipid and fattyacid analysis of fresh and frozen-thawed immature and in vitro-matured bovine oocytes. Reproduction 122(1), 131-138. doi:10.1530/REP.0. 1220131

Kruip, T. A. M., Cran, D. G., Van Beneden, T. H., and Dieleman, S. J. (1983). Structural changes in bovine oocytes during final maturation in vivo. Gamete Res. 8(1), 29-47. doi:10.1002/MRD.1120080105

Kuerschner, L., Moessinger, C., and Thiele, C. (2008). Imaging of lipid biosynthesis: how a neutral lipid enters lipid droplets. Traffic 9(3), 338-352. doi:10.1111/J.1600-0854.2007.00689.X

Leibfried-Rutledge, M. L., Critser, E. S., Eyestone, W. H., Northey, D. L., and Fiste, N. L. (1987). Development potential of bovine oocytes matured in vitro and in vivo. Biol. Reprod. 36, 376-383. doi:10.1095/ BIOLREPROD36.2.376

Leroy, J. L., Vanholder, T., Mateusen, B., Christophe, A., Opsomer, G., de Kruif, A., Genicot, G., and Van Soom, A. (2005). Non-esterified fatty acids in follicular fluid of dairy cows and their effect on developmental capacity of bovine oocytes in vitro. Reproduction 130(4), 485-495. doi:10.1530/REP.1.00735

Paczkowski, M., Silva, E., Schoolcraft, W. B., and Krisher, R. L. (2013). Comparative importance of fatty-acid beta-oxidation to nuclear maturation, gene expression and glucose metabolism in mouse, bovine and porcine cumulus-oocyte complexes. Biol. Reprod. 88(5), 111. doi:10.1095/BIOLREPROD.113.108548

Rizos, D., Fair, T., Papadopoulos, S., Boland, M. P., and Lonergan, P. (2002). Developmental, qualitative and ultrastructural differences between ovine and bovine embryos produced in vivo or in vitro. Mol. Reprod. Dev. 62(3), 320-327. doi:10.1002/MRD.10138
Rizos, D., Gutiérrez-Adán, A., Pérez-Garnelo, S., de la Fuente, J., Boland, M. P., and Lonergan, P. (2003). Bovine embryo culture in the presence or absence of serum: implications for blastocyst development, cryotolerance and messenger RNA expression. Biol. Reprod. 68(1), 236-243. doi:10.1095/BIOLREPROD.102.007799

Romek, M., Gajda, B., Rolka, M., and Smorag, Z. (2011). Mitochondrial activity and morphology in developing porcine oocytes and preimplantation non-cultured and cultured embryos. Reprod. Domest. Anim. 46(3), 471-480. doi:10.1111/J.1439-0531.2010.01691.X

Russell, D. F., Baqir, S., Bordignon, J., and Betts, D. H. (2006). The impact of oocyte maturation media on early bovine embryonic development. Mol. Reprod. Dev. 73(10), 1255-1270. doi:10.1002/MRD.20553

Sata, R., Tsujii, H., Abe, H., Yamashita, S., and Hoshi, H. (1999). Fatty-acid composition of bovine embryos cultured in serum-free and serumcontaining medium during early embryonic development. J. Reprod. Dev. 45(1), 97-103. doi:10.1262/JRD.45.97

Stone, S. J., Levin, M. C., Zhou, P., Han, J. Y., Walther, T. C., and Farese, R. V. (2009). The endoplasmic reticulum enzyme DGAT2 is found in mitochondria-associated membranes and has a mitochondrial targeting signal that promotes its association with mitochondria. J. Biol. Chem. 284(8), 5352-5361. doi:10.1074/JBC.M805768200

Tarazona, A. M., Rodriguez, J. I., Restrepo, L. F., and Olivera-Angel, M. (2006). Mitochondrial activity, distribution and segregation in bovine oocytes and in embryos produced in vitro. Reprod. Domest. Anim. 41(1), 5-11. doi:10.1111/J.1439-0531.2006.00615.X

Tsujii, H., Yahia Khandoker, M. A. M., and Hamano, K. (2001). Lipid in mammalian embryo development. J. Mamm. Ova Res. 18(3), 73-80. doi:10.1274/JMOR.18.73

Van Blerkom, J. (2011). Mitochondrial function in the human oocyte and embryo and their role in developmental competence. Mitochondrion 11(5), 797-813. doi:10.1016/J.MITO.2010.09.012 